Research Paper

\title{
In-hospital Mortality after Surgical Resection in Hepatocellular Carcinoma Patients with Portal Vein Tumor Thrombus
}

\author{
Xiu-Ping Zhang1 ${ }^{*}$, Yu-Zhen Gao ${ }^{*}$, Zhen-Hua Chen ${ }^{1 *}$, Kang Wang1, Yu-Qiang Cheng1, Wei-Xing Guo', Jie \\ Shi $^{1}$, Cheng-Qian Zhong 3 , Fan Zhang ${ }^{4}$, Shu-Qun Cheng ${ }^{1 \times}$ \\ 1. Department of Hepatic Surgery VI, Eastern Hepatobiliary Surgery Hospital, Second Military Medical University. Shanghai, China; \\ 2. Department of Molecular Diagnosis, Clinical Medical School, Yangzhou University, Yangzhou, China. \\ 3. LongYan First Hospital, Affiliated to Fujian Medical University, FuJian, China. \\ 4. Department of Hepatic Surgery, BinZhou medical University Hospital, BinZhou, China. \\ *Xiu-Ping Zhang, Yu-Zhen Gao and Zhen-Hua Chen contributed equally to this manuscript. \\ $\square$ Corresponding author: Shu-Qun Cheng, MD, Eastern Hepatobiliary Surgery Hospital, Second Military Medical University, 225 Changhai Road, Shanghai \\ 200433 China. Email: chengshuqun@aliyun.com \\ (c) Ivyspring International Publisher. This is an open access article distributed under the terms of the Creative Commons Attribution (CC BY-NC) license \\ (https://creativecommons.org/licenses/by-nc/4.0/). See http://ivyspring.com/terms for full terms and conditions.
}

Received: 2018.05.05; Accepted: 2018.10.03; Published: 2019.01.01

\begin{abstract}
Background: Survival benefit of surgical resection for hepatocellular carcinoma (HCC) patients with portal vein tumor thrombus (PVTT) has been approved recently. However, risk factors for in-hospital mortality in these patients remain unclear. We aimed to determine risk factors and reduce the mortality of these patients.

Methods: We analyzed data for 521 of all 1531 HCC patients with PVTT underwent surgery. The primary outcome measure was in-hospital mortality after surgical resection. Univariate and Multivariate cox-regression were performed to identify independent predictors of in-hospital mortality. The methods of Kaplan-Meier, bootstrap and ten-fold-cross validation were applied to validate the risk factors.

Results: 521 of 1531 patients in 2004-2012 occurred for the diagnosis of HCC associated with PVTT and underwent surgical resection as a training cohort. Other 325 patients in 2013-2016 were included as a validation cohort. Overall mortality of postoperative in-patients was 3.3\% (17/521) and $2.8 \%$ (9/325), respectively. Univariate analysis of mortality revealed that frequency of hospitalization, total albumin, different types of PVTT, bleeding volume, blood transfusion, resection volume, and tumor volume were related with mortality. Therefore, the bootstrap validation reflected that the risk factors of multivariate cox regression in modell (frequency of hospitalization, bleeding volume, and tumor volume) and model 2 (frequency of hospitalization, bleeding volume and total albumin) were stable with mortality in hospital. Ten-fold cross-validation of cox regression analysis showed that the mean C-statistic with $95 \% \mathrm{Cl}$ of modell and model 2 respectively were $0.887(0.779-0.976)$ and $0.867(0.789-0.966)$ for predicting in-hospital mortality. Consistency results of models were in the training cohort and validation cohort.

Conclusion: Total albumin, tumor volume, intraoperative bleeding and frequency of hospitalization were independent predictive factors for in-hospital mortality in HCC patients with PVTT under surgery. Further study is warranted to utilize these factors to lower in-hospital mortality.
\end{abstract}

Key words: surgical resection, hepatocellular carcinoma, portal vein tumor thrombus, in-hospital mortality

\section{Introduction}

Hepatocellular carcinoma (HCC) is the fifth most common malignancy and the third leading cause of tumor-related death in the world [1]. Although multiple treatments, such as surgical resection, liver transplantation, transarterial chemoembolization (TACE) and radiotherapy (RT), could be performed for selected HCC patients, the high recurrence rate has impaired long-time survival. Indeed, HCC 
patients associated with portal vein tumor thrombus have been reported to have an extremely poor prognosis[2]. PVTT has been reported to be an independent factor predicting high recurrence and poor survival rate [3-5]. The median survival time (MST) of HCC patients with PVTT was found to be significantly reduced (2-4 months) compared to patients without PVTT [6]. According to the Barcelona Clinic for Liver Cancer (BCLC) Staging System and treatment guidelines, PVTT was regarded as a contraindication of operation in HCC patients[1, 7]. Furthermore, sorafenib was only proposed treatment option for these patients with PVTT, with MST of as short as 10.7 months [8]. However, the Asia-Pacific guideline recommends multidisciplinary treatment of surgery, TACE, RT and sorafenib as treatment options [9-11]. Among these, surgical resection may be only completely cure HCC and play beneficial role in the treatment of some selected patients with PVTT [12].

Aggressive surgical resection for HCC with PVTT been proposed by several tertiary centers, concluding that surgical resection was associated with a longer survival outcome than non-surgical treatment for some selected HCC patients with PVTT [12-14]. Because of advances in surgical techniques and perioperative management, surgical resection has become a reasonably recognized treatment option with an acceptable mortality and morbidity rate with a better survival benefits [2,11-13, 15, 16]. Based on recent studies, in-hospital mortality after surgical resection in HCC patients with PVTT ranged from $3.7 \%$ to $10 \%$ [12, 17-19]. Perioperative management and skilled surgical operation counted a great deal to reduce the mortality rate by lowering risk factors of these patients. However, the independent risk factors of in-hospital mortality for HCC patients with PVTT after surgical resection is still unclear.

The aim of the present study was to determine the risk factors for in-hospital mortality in HCC patients with PVTT after surgical resection. Further, these conclusions may provide surgeon with valuable clinical reference to lowering in-hospital mortality of HCC patients with PVTT under surgical resection aiming of prolonging these patients' survival.

\section{Materials and Methods}

\section{Diagnostic Criteria for different types of PVTT}

PVTT was diagnosed using imaging examination (ultrasound, CT and MRI) and/or intraoperative and postoperative histopathology [20] in HCC patients. According to Cheng's Classification, PVTT was classified into 4 types based on the extent of PVTT in the portal vein: Type I, tumor thrombus in the segmental branches of the portal vein or above;
Type II, tumor thrombus extending to the right or the left portal vein; Type III, tumor thrombus extending to the main portal vein; and Type IV, tumor thrombus extending to the main portal vein and the superior mesenteric vein.

\section{Study population and inclusion criteria}

This study was conducted retrospectively in Eastern Hepatobiliary Surgery Hospital (EHBH) and approved by the Institutional Ethics Committee of EHBH. Written informed consent was obtained from all the patients for their data to be used for study. The primary measured outcome was in-hospital mortality after surgical resection, defined as death prior to discharge regardless of cause or time from operation. The inclusion criteria were: (1) HCC patients with PVTT diagnosed by diagnostic criteria mentioned above. (2) HCC patients with PVTT performed under surgical resection with Child-Pugh class A or selected B liver function. (3) Patients without macroscopic hepatic vein tumor thrombus, extrahepatic spread or distant metastases and no other malignancies. (4) Patients without other treatments such as TACE, chemotherapy, radiotherapy and sorafenib. In-hospital mortality of HCC patients with PVTT.

\section{Surgery Procedures}

The surgical procedures have been reported in previous study[21]. Only HCC patients with PVTT with Child-Pugh A or selected B liver function were offered hepatic resection $[4,22,23]$. Surgical resection was performed under general anesthesia using a right subcostal incision with a midline extension. Intraoperative ultrasound was routinely performed. Thrombectomy was performed according to the different types of PVTT. When the lesion is resectable, HCC patients with type I and II PVTT was recommended be performed surgical resection of the PVTT en bloc with the primary HCC. Depending on the patient's preference, partial hepatectomy combined with thrombectomy or main portal vein resection followed by reconstruction may be performed for some selected HCC patients with type III and IV PVTT. Type III and IV PVTT was extracted out from the opened stump of the portal vein if the PVTT protruded into the main portal vein beyond the resection line. If the PVTT extended into the main portal trunk and its primary branches on both sides, the main portal trunk was exposed and was clamped distal to the PVTT. The PVTT was extracted after an incision was made at the bifurcation of the right and left portal veins. After flushing with normal saline and confirming that no PVTT remained, the stump was closed by a continuous suture. 


\section{Statistical Analysis}

In our queue cohorts, the continuity variables are tested by normality. Normality data are represented as mean \pm standard deviation and an independent- $t$ test was used to test the difference between two groups. No-Normality data are represented as median and the interquartile range, and a Mann-Whitney U-test as used to compare sets of continuous variables. Counting data are presented as ratio. We use the bootstrap method to calculate the $95 \%$ interval. In addition, we stratified the patients into four groups according to value of quarter in the different continuity variables for further statistical analysis. Univariate and multivariate cox regression were used to explore the independent factors for the death in-hospital HCC patients with PVTT. We established model 1 and model 2 and calculated their C-statistics with 95\% CI respectively for predicting in-hospital mortality, because of the particularity of indicators. The mortality rates during hospitalization of indicators with different stratification were presented by Kaplan-Meier curve, and the difference was tested by log-rank test. The same statistical operation was implemented in the validation group. Then, the methods of bootstrap for the coefficients of risk factors were preformed to validate the stable results of cox regression in different models. Finally, the ten-fold-cross-validation was carried by using the training and internal validation cohort data. All statistical methods are carried out in SPSS (SPSS.inc, USA, version 24.0) and R(Foundation for Statistical Computing, Austria, version 3.4.4), and P-value is less than 0.05 with statistical significance.

\section{Results}

\section{Baseline Characteristics of Patients}

From 2004 to 2012 in EHBH, 1531 patients were diagnosed as HCC with PVTT. 521 of 1531patients under surgical treatment included in our study were regarded as a training cohort. Among them, only 17 patients were dead during hospitalization after surgical treatment, with the in-hospital mortality of $3.3 \%(17 / 521)$. In addition, from 2013 to 2016 in $\mathrm{EHBH}, 325$ patients with HCC and PVTT underwent the surgical treatment were including and regarded as the validation cohort with the in-hospital mortality of $2.8 \%(9 / 325)$. The flow chart of the including HCC patients with PVTT was shown in Figure 1. Baseline and clinical characteristics of enrolled HCC patients with PVTT in two cohorts were listed in Table 1. Although they were not included in the same time, there were no significant differences in most variables between two cohorts. All HCC patients with PVTT were under surgical treatment with Child-Pugh A or selected B liver function, resulting from perioperative management. More men than women with HCC and PVTT were included in the analysis, with median 48 and 49 years old, in the training /internal validation cohort.

Table 1. Clinical characteristics of the study populations who with HCC and PVTT after resection.

\begin{tabular}{|c|c|c|c|c|}
\hline Variables & $\begin{array}{l}2004-2012 \\
\text { cohort(n=521) }\end{array}$ & $\begin{array}{l}2013-2016 \\
\text { cohort }(n=325)\end{array}$ & $\mathrm{X}^{2} / \mathrm{Z}$ & $\mathbf{P}$ \\
\hline Age, year & $49.0(42.0-56.0)$ & $48.0(40.0-55.5)$ & -0.771 & 0.441 \\
\hline Gender & & & 0.001 & 0.978 \\
\hline Male & $470(90.21 \%)$ & $293(90.15 \%)$ & & \\
\hline Female & $51(9.79 \%)$ & $32(9.85 \%)$ & & \\
\hline $\begin{array}{l}\text { Time of hospitalization, } \\
\text { days }\end{array}$ & $15.0(13.0-20.0)$ & 14.1(13.0-19.0) & -0.264 & 0.792 \\
\hline \multicolumn{3}{|c|}{ Postoperative hospitalization } & 0.164 & 0.686 \\
\hline Alive & $504(96.74 \%)$ & $316(97.23 \%)$ & & \\
\hline Dead & $17(3.26 \%)$ & $9(2.77 \%)$ & & \\
\hline \multicolumn{3}{|l|}{ Number of hospitalization } & 0.181 & 0.671 \\
\hline First & $120(23.03 \%)$ & $79(24.31 \%)$ & & \\
\hline Again & $401(76.97 \%)$ & $246(75.69 \%)$ & & \\
\hline Child-Pugh grade & & & 0.167 & 0.683 \\
\hline A & $511(98.08 \%)$ & $320(98.46 \%)$ & & \\
\hline B & $10(1.92 \%)$ & $5(1.54 \%)$ & & \\
\hline \multicolumn{5}{|c|}{ Preoperative serological Indexes } \\
\hline HBsAg,yes & $463(88.9 \%)$ & $280(86.2 \%)$ & 1.378 & 0.240 \\
\hline Total albium,mg/ml & $72.0(68.2-77.0)$ & $76.0(68.6-79.0)$ & -0.310 & 0.757 \\
\hline ALT, U/L & $45.0(29.4-66.0)$ & $38.0(29.1-66.6)$ & -0.092 & 0.227 \\
\hline AST, U/L & $49.0(34.0-67.0)$ & $39.0(24.0-68.5)$ & -0.180 & 0.157 \\
\hline $\mathrm{AFP}, \mathrm{ng} / \mathrm{mL}$ & $\begin{array}{l}1000.0(82.1-1210 . \\
0)\end{array}$ & $\begin{array}{l}914.0(95.9-1210.0 \\
)\end{array}$ & -0.964 & 0.343 \\
\hline $\mathrm{CEA}, \mathrm{ng} / \mathrm{mL}$ & $2.2(1.5-3.3)$ & 2.8(1.4-3.3) & -0.310 & 0.757 \\
\hline CA199, ng/mL & $23.8(13.6-40.3)$ & $20.2(14.1-40.4)$ & -0.258 & 0.797 \\
\hline \multicolumn{5}{|l|}{ Intraoperative Indexes } \\
\hline Thrombectomy, yes & $261(50.10 \%)$ & $164(50.46 \%)$ & 0.011 & 0.918 \\
\hline Cholecystectomy, yes & $456(87.52 \%)$ & $284(87.38 \%)$ & 0.004 & 0.953 \\
\hline Ascites, yes & $60(11.52 \%)$ & $35(10.77 \%)$ & 0.112 & 0.738 \\
\hline Blood Transfusion, yes & $162(31.1 \%)$ & $97(29.8 \%)$ & 0.147 & 0.702 \\
\hline Bleeding, $100 \mathrm{ml}$ & $4.0(2.5-8.0)$ & $4.0(2.6-8.0)$ & -0.266 & 0.790 \\
\hline PVTT,type & & & 0.583 & 0.900 \\
\hline I & $134(25.72 \%)$ & $84(25.85 \%)$ & & \\
\hline II & $274(52.59 \%)$ & $169(52.00 \%)$ & & \\
\hline III & $108(20.73 \%)$ & $67(20.62 \%)$ & & \\
\hline IV & $5(0.96 \%)$ & $5(1.54 \%)$ & & \\
\hline Metastasis, yes & $48(9.21 \%)$ & $28(8.62 \%)$ & 0.087 & 0.767 \\
\hline \multicolumn{5}{|l|}{ Pathological Indexes } \\
\hline Liver cirrhosis,yes & $377(72.36 \%)$ & $234(72.00 \%)$ & 0.013 & 0.909 \\
\hline Number of tumor, Multi & $63(12.09 \%)$ & $48(14.77 \%)$ & 1.258 & 0.262 \\
\hline Vascular invasion,yes & $443(85.03 \%)$ & $273(84.00 \%)$ & 0.163 & 0.687 \\
\hline Resection Volume, $\mathrm{cm}^{3}$ & 137.8(97.9-194.4) & 145.0(87.1-198.6) & -0.209 & 0.835 \\
\hline Tumor Volume, $\mathrm{cm}^{3}$ & $52.2(28.0-93.0)$ & $58.6(28.6-95.4)$ & -0.366 & 0.714 \\
\hline
\end{tabular}

NA: not available

\section{The relationship between the type of PVTT and the in-hospital mortality in training cohort}

The HCC patients with different types of PVTT were under surgical treatment had entirely distinct prognosis [12, 14, 23]. Type I and type II PVTT patients had a better prognosis than those with type III and IV [14]. We further clarified the relationship between the different type of PVTT and the in-hospital mortality of HCC patients with PVTT after surgical resection. The survival curve of different type 


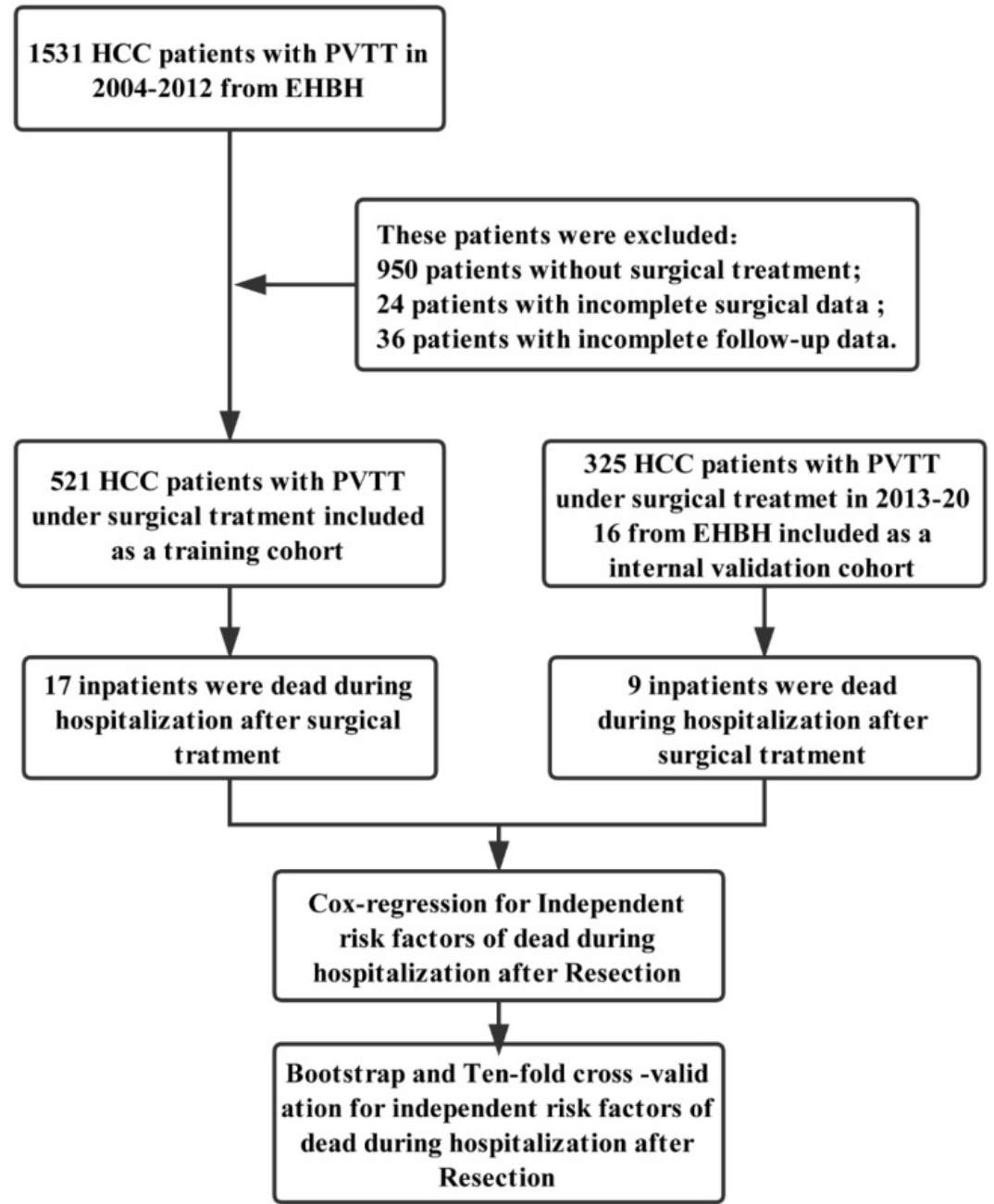

Figure 1. The flow chart of screening all HCC patients for selected patients with PVTT under surgical resection in 2004-2012 and 2013-2016.

of PVTT patients was showed in Figure 2A. HCC patients with type I PVTT had a longer survival than those with type IV. Contrarily, type IV patients had high in-hospital mortality than type I PVTT patients.

Figure $2 \mathrm{~B}$ was the life table of the HCC patients with different type of PVTT under surgery in each 5 days during the time of hospitalization. In Figure 2B, the death of PVTT patients under surgery was almost up to 30 days of hospitalization. In-hospital mortality of patients undergoing surgery with type I, II, III, IV PVTT was $1.5 \%$ (2/134), 3.3\% (9/274), 3.7\% (4/108), and $40 \%(2 / 5)$, respectively. From the above, the different type of PVTT has a significant relationship with the in-hospital mortality in the HCC patients after resection.

\section{Univariate analysis of in-hospital mortality for HCC patients with PVTT after surgery in training cohort}

The univariate analysis had revealed that eight potentially potential risk factors could be related with the in-hospital in the HCC patients with PVTT after surgical resection. These risk factors belong to different clinical categories, respectively were History of hospitalization: Frequency of hospitalization (HR,95\% CI $=0.057$ (0.016-0.202),P<0.001), Preoperative Serological examination: the expression of total albumin (HR,95\% CI=1.612 (1.004-2.587), $\mathrm{P}=0.048)$; Intraoperative index: the type of PVTT (IV vs I, HR, 95\% CI=66 (5.949-732.281), $\mathrm{P}<0.001), \quad$ Intraoperative Bleeding volume (HR,95\%CI=1.086 (1.044-1.129), $\mathrm{P}<0.001)$, Blood Transfusion (HR,95\%CI=5.664 (1.96116.358), $\mathrm{P}=0.001) ;$ Pathological Indexes; Resection Volume (HR, 95\% CI=1.134 (1.004-1.281), $\mathrm{P}=0.043)$, Tumor Volume (HR, 95\%CI=1.006 (1-1.011), $\mathrm{P}=0.035)$ and Vascular invasion $(\mathrm{HR}, 95 \% \mathrm{CI}=0.27$ (0.079-0.927), $\mathrm{P}=0.037$ ). As the results, preoperative liver function (Total Alb), types of PVTT, the operation (intraoperative bleeding, resection volume, tumor volume) were risk factors for in-hospital mortality for HCC patients with PVTT after surgery. The other details were shown in Table 2. 
A Hazard Function

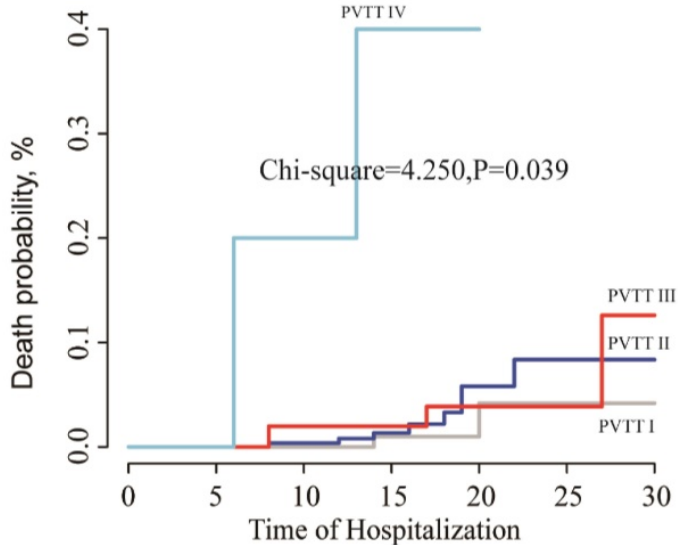

B Life table

The number at risk with censors of different types of PVTT patients in-hospital.

\begin{tabular}{lccccccc}
\hline Type & 0 & 5 & 10 & 15 & 20 & 25 & 30 \\
\hline PVTT I & $134(0)$ & $132(0)$ & $124(1)$ & $82(0)$ & $31(0)$ & $10(0)$ & $4(0)$ \\
PVTT II & $274(1)$ & $272(1)$ & $260(6)$ & $140(0)$ & $62(0)$ & $24(0)$ & $10(0)$ \\
PVTT III & $108(2)$ & $104(2)$ & $97(1)$ & $64(1)$ & $36(0)$ & $14(0)$ & $6(0)$ \\
PVTT IV & $5(1)$ & $5(0)$ & $4(1)$ & $2(0)$ & $2(0)$ & $2(0)$ & $2(0)$
\end{tabular}

Note:the number of total and deaths patients was

respectively in the upper and lower cells at each 5 days

Figure 2. A: The survival curve of four types of PVTT in 521 HCC patients; B: The Life table: the number of total and the death patients at each 10 days during the period of hospitalization.

A. Frequency of hospitalization

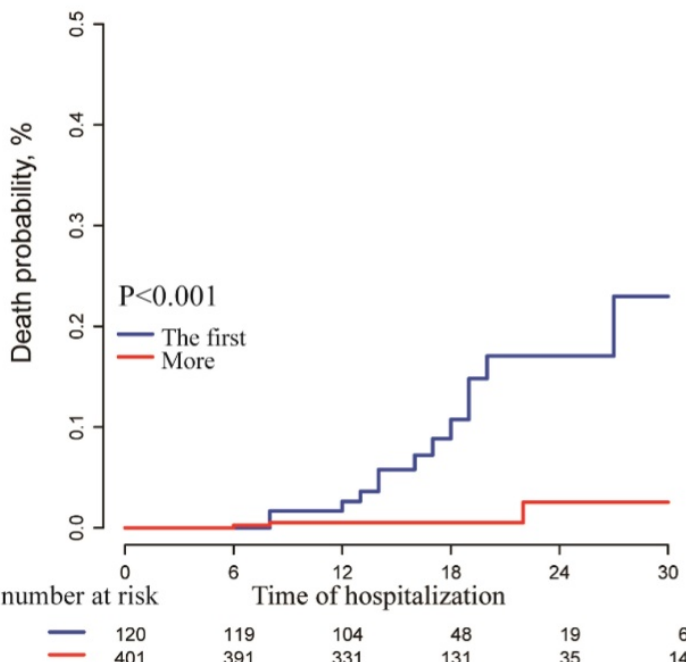

C. Tumor volume,quarter

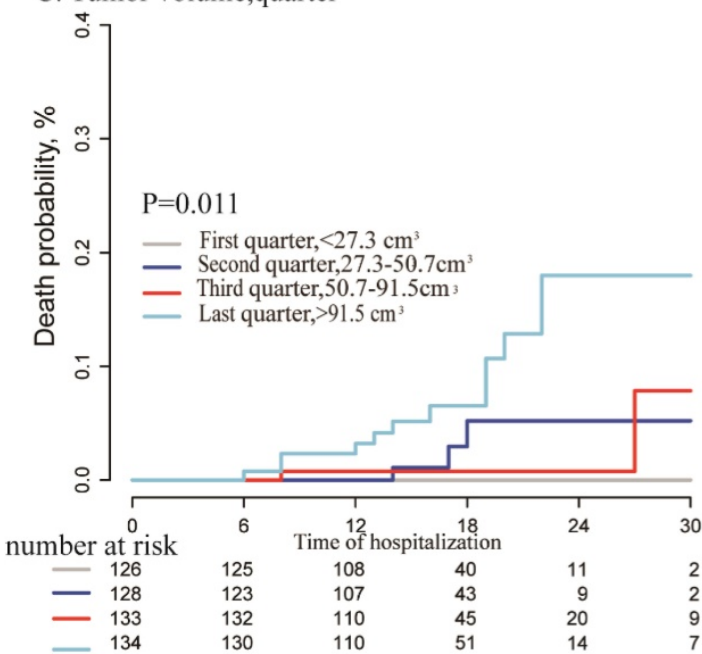

B. Intraoperative bleeding,quarter

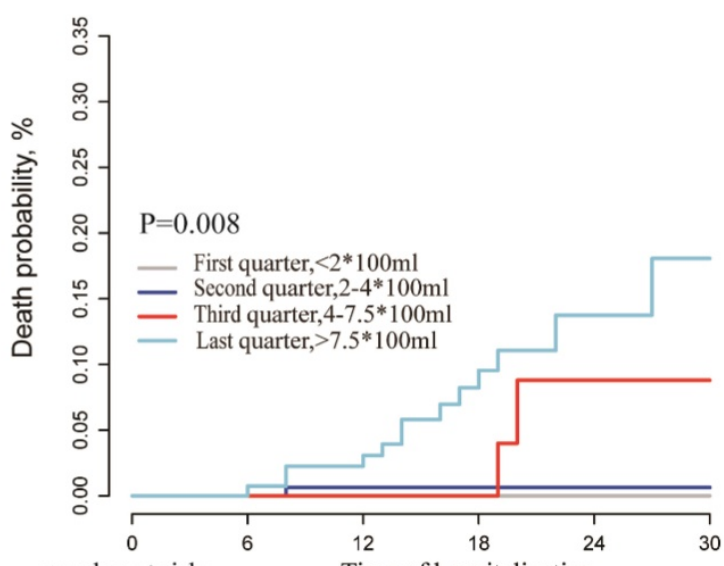

number at risk

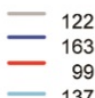

119
158
99
134

Time of hospitalization

D. Total albumin,quarter

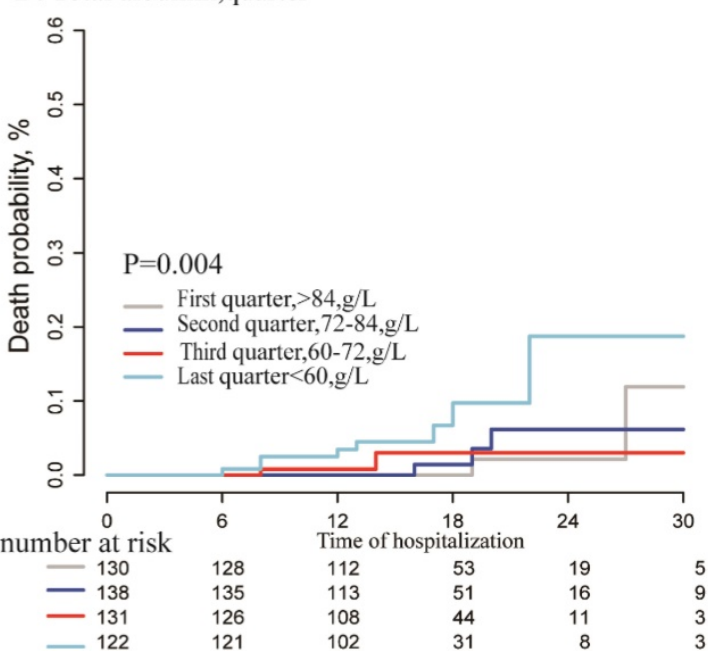

Figure 3. Kaplan-Meier analysis for different variables: A: Frequency of hospitalization(first vs more ,P<0.001);B: intraoperative bleeding volume (Per quarter, $P=0.008$ ); $C$ : tumor volume (per quarter, $P=0.011$ ), $D$ : total albumin (per quarter, $P=0.004$ ) 
Table 2. Univariate analysis of mortality for HCC patients with PVTT (all resections).

\begin{tabular}{|c|c|c|c|c|}
\hline Variables & B & $\mathbf{P}$ & HR & $95 \% \mathrm{CI}$ \\
\hline Gender, male vs female & 0.711 & 0.277 & 2.036 & $0.565-7.336$ \\
\hline Age, per year & 0.028 & 0.266 & 1.028 & $0.979-1.08$ \\
\hline Time of hospitalization & -0.004 & 0.925 & 0.996 & $0.926-1.071$ \\
\hline $\begin{array}{l}\text { Frequency of hospitalization, } \\
\text { first vs more }\end{array}$ & -2.863 & $<0.001$ & 0.057 & $0.016-0.202$ \\
\hline \multicolumn{5}{|c|}{ Preoperative serological examination } \\
\hline HBsAg, positive vs negative & -0.519 & 0.426 & 0.595 & $0.166-2.134$ \\
\hline Total Alb, per quarter, g/L & 0.478 & 0.048 & 1.612 & $1.004-2.587$ \\
\hline $\mathrm{ALT}$, per quarter, $\mathrm{U} / \mathrm{L}$ & 0.391 & 0.098 & 1.478 & $0.93-2.347$ \\
\hline AST, per quarter, $\mathrm{U} / \mathrm{L}$ & 0.303 & 0.18 & 1.354 & $0.869-2.11$ \\
\hline $\mathrm{AFP}$, per $\mathrm{ug} / \mathrm{ml}$ & 0.725 & 0.117 & 2.064 & $0.834-5.112$ \\
\hline CEA, per quarter, $\mathrm{ng} / \mathrm{mL}$ & -0.061 & 0.781 & 0.94 & $0.609-1.452$ \\
\hline CA199, per quarter, ng/mL & 0.321 & 0.164 & 1.378 & $0.878-2.165$ \\
\hline \multicolumn{5}{|c|}{ Intraoperative and Pathological Index } \\
\hline PVTT (Ref,I) & & 0.006 & & \\
\hline II & 0.913 & 0.243 & 2.491 & $0.538-11.53$ \\
\hline III & 0.634 & 0.492 & 1.886 & $0.309-11.493$ \\
\hline IV & 4.19 & 0.001 & 66 & $5.949-732.281$ \\
\hline Ascites, yes vs no & 0.519 & 0.426 & 1.68 & $0.469-6.026$ \\
\hline $\begin{array}{l}\text { Intraoperative bleeding, per } \\
\text { quarter }\end{array}$ & 0.082 & $<0.001$ & 1.086 & $1.044-1.129$ \\
\hline Blood Transfusion, yes vs no & 1.734 & 0.001 & 5.664 & $1.961-16.358$ \\
\hline Resection Volume, per quarter & 0.126 & 0.043 & 1.134 & $1.004-1.281$ \\
\hline Tumor Volume, per quarter & 0.006 & 0.035 & 1.006 & $1-1.011$ \\
\hline Number of tumor, one vs more & 0.842 & 0.152 & 2.321 & $0.733-7.352$ \\
\hline Liver cirrhosis, no vs yes & 0.506 & 0.348 & 1.659 & $0.576-4.78$ \\
\hline Capsular invasion, no vs yes & 0.296 & 0.309 & 1.345 & $0.76-2.38$ \\
\hline
\end{tabular}

\section{Multivariate and Kaplan-Meier analysis of in-hospital mortality for HCC patients with PVTT after surgery in training cohort}

In multivariate analysis, we explored the independent risk factors for hospitalized HCC patients with PVTT after resection to build two cox regression models for multivariate analysis, because of the high correlation between liver resection volume and tumor volume. The factors of tumor volume (HR, 95\% CI=2.37 (1.191-4.716), $\mathrm{P}=0.014)$; Intraoperative bleeding volume, $(\mathrm{HR}, 95 \% \mathrm{CI}=3.993$ (1.373 -11.612), $\mathrm{P}=0.011)$ and Frequency of hospitalization (HR,95\% CI=-2.174,0.114 (0.031-0.416), $\mathrm{P}=0.001)$ showed been the significant factors in the first model which contain the index of tumor volume. In the second model, we unexpectedly found that the Resection volume was not an independent risk factor for HCC patients with PVTT in-hospital (HR, 95\%CI= 1.001 (0.995 -1.006), $\mathrm{P}=0.82)$, however, the factor of total albumin (HR, 95\% CI=1.649 (1.014-2.681), $\mathrm{P}=0.044)$ was existed in the second model. More details were shown in Table S1. Kaplan-Meier analysis was to analyses the above independent risk factors in the two-cox regression model as shown in Figure 3. From these results, HCC and PVTT patients under surgery with more frequency of hospitalization $(\mathrm{P}<0.001)$, more intraoperative bleeding $(\mathrm{P}=0.008)$, more tumor volume $(\mathrm{P}=0.011)$, and lower total albumin $(\mathrm{P}=0.004)$ had a higher in-hospital mortality after surgical resection.

Table 3. Ten-fold cross-validation multivariable cox regression on "death in hospitalization" in the training/internal validation cohort.

\begin{tabular}{|c|c|c|c|c|c|c|c|c|c|c|c|}
\hline \multirow[t]{2}{*}{ Variables } & \multicolumn{10}{|c|}{ B Coefficients } & \multirow{2}{*}{$\begin{array}{l}\text { B-coefficients- } \\
\text { Mean } \pm S D\end{array}$} \\
\hline & $\overline{1}$ & 2 & 3 & 4 & 5 & 6 & 7 & 8 & 9 & 10 & \\
\hline $\begin{array}{l}\text { Frequency of hospitalization, one vs more } \\
\text { PVTT, type }\end{array}$ & -2.510 & -2.691 & -1.802 & -1.833 & -2.473 & -2.562 & -3.901 & -2.072 & -1.530 & -2.282 & $-2.366 \pm 0.661$ \\
\hline I & Ref & Ref & Ref & Ref & Ref & Ref & Ref & Ref & Ref & Ref & \\
\hline II & -0.700 & 0.466 & -0.159 & 0.898 & -0.602 & -0.530 & 0.240 & -0.483 & -0.105 & -0.350 & $-0.133 \pm 0.520$ \\
\hline III & -0.414 & 0.168 & 0.403 & 0.494 & -0.757 & -2.047 & 0.255 & -0.066 & -0.272 & -0.586 & $-0.282 \pm 0.750$ \\
\hline IV & 1.680 & 2.872 & 2.895 & 4.113 & -12.223 & 0.428 & 7.095 & 1.799 & 2.147 & 1.866 & $1.267 \pm 5.072$ \\
\hline Intraoperative bleeding, per quarter, $100 \mathrm{ml}$ & 1.134 & 1.203 & 2.456 & 2.069 & 1.096 & 1.337 & 1.377 & 1.796 & 1.431 & 1.175 & $1.507 \pm 0.455$ \\
\hline Tumor volume, per quarter, $\mathrm{cm}^{3}$ & 1.000 & 0.689 & 0.497 & 0.302 & 0.955 & 1.006 & 1.677 & 0.783 & 1.013 & 1.629 & $0.955 \pm 0.437$ \\
\hline Total albiumn, per quarter\# & 0.327 & 0.315 & 0.433 & 0.608 & 0.288 & 0.837 & 0.738 & 0.603 & 0.424 & 0.530 & $0.510 \pm 0.186$ \\
\hline Intraoperative Blood Transfusion, yes vs no & -1.067 & -1.040 & -1.271 & -1.236 & -1.591 & -0.432 & -0.349 & $-1.432)$ & -0.944 & -1.229 & $-1.059 \pm 0.400$ \\
\hline C-statistic for model1 & 0.878 & 0.891 & 0.887 & 0.799 & 0.89 & 0.886 & 0.928 & 0.877 & 0.877 & 0.877 & $0.887(0.779-0.976)$ \\
\hline $\begin{array}{l}\text { Frequency of hospitalization, one vs more } \\
\text { PVTT, type }\end{array}$ & -2.437 & -2.721 & -2.050 & -2.154 & -2.374 & -2.341 & -3.250 & -1.990 & -1.575 & -2.182 & $-2.307 \pm 0.450$ \\
\hline I & Ref & Ref & Ref & Ref & Ref & Ref & Ref & Ref & Ref & Ref & \\
\hline II & -0.594 & 0.657 & 0.071 & 0.673 & -0.293 & -0.342 & 0.335 & -0.059 & 0.256 & -0.069 & $0.064 \pm 0.420$ \\
\hline III & -0.139 & 0.278 & 0.684 & 0.393 & -0.465 & -1.737 & 0.165 & 0.344 & 0.019 & -0.192 & $-0.065 \pm 0.674$ \\
\hline IV & 2.024 & 3.794 & 4.241 & 4.729 & -11.763 & 1.189 & 6.947 & 2.123 & 2.652 & 2.756 & $1.869 \pm 5.067$ \\
\hline Intraoperative bleeding, per quarter, $100 \mathrm{ml}$ & 1.049 & 1.211 & 2.430 & 2.076 & 0.989 & 0.968 & 1.201 & 1.513 & 1.364 & 1.132 & $1.393 \pm 0.490$ \\
\hline Total albiumn, per quarter\# & 0.380 & 0.312 & 0.372 & 0.665 & 0.692 & 0.886 & 0.401 & 0.589 & 0.556 & 0.598 & $0.505 \pm 0.187$ \\
\hline Resection volume, per quarter, $\mathrm{cm} 3$ & 0.402 & 0.266 & 0.535 & 0.517 & 0.305 & 0.504 & 0.408 & 0.594 & 0.499 & 0.881 & $0.491 \pm 0.172$ \\
\hline Intraoperative Blood Transfusion, yes vs no & -0.949 & -0.952 & -1.371 & -1.429 & -1.069 & -0.146 & -0.229 & -1.235 & -0.816 & -1.171 & $-0.937 \pm 0.439$ \\
\hline C-statistic for model2 & 0.846 & 0.857 & 0.89 & 0.758 & 0.888 & 0.851 & 0.867 & 0.849 & 0.849 & 0.849 & $0.867(0.789-0.966)$ \\
\hline Sample size of the training cohort (no.) & 406 & 409 & 411 & 412 & 405 & 408 & 415 & 418 & 414 & 419 & - \\
\hline Sample size of the testing cohort (no.) & 110 & 110 & 110 & 112 & 109 & 112 & 109 & 105 & 108 & 102 & - \\
\hline
\end{tabular}




\section{Validation for risk factors in-hospital mortality for HCC patients with PVTT after surgery}

Bootstrap method for cox-regression showed that all the coefficient of risk factors had a statistical significance in the training cohort. More details were shown in Table S2. In addition, the 10-fold-cross validation was also used to validate the coefficient of risk factors by using the training data and internal validation data and it showed that the variables were statistically significant in the model 1 and model 2 with cox-regression in most times. Therefore, the mean C-statistic with $95 \% \mathrm{CI}$ of model 1 and model 2 respectively were $0.887(0.779-0.976)$ and $0.867(0.789$ 0.966) for predicting in-hospital mortality. Details were shown in Table 3. Finally, Kaplan-Meier curve analysis was also performed in the validation cohort, as it showed in Figure S1. The risk factors of frequency of hospitalization $(\mathrm{P}=0.001)$, intraoperative bleeding $(\mathrm{P}=0.016)$ and total albumin $(\mathrm{P}=0.008)$ were also related with in-hospital mortality, except tumor volume $(\mathrm{P}=0.051)$. Details were shown in Figure S1. In summary, total albumin, tumor volume, intraoperative bleeding, frequency of hospitalization could be regarded as the independent risk factors for in-hospital mortality in HCC patients with PVTT under surgical resection.

\section{Discussion}

Previous studies with extensive evidence based on the safety and efficacy of HR for HCC patients with PVTT showed that patients with first-order branch PVTT and preserved liver function had a better prognosis under HR.[24, 25]. However, in-hospital mortality rate, which ranged from $3.7 \%$ to $10 \%$, had been reported for HCC patients with PVTT after surgery[13]. Few studies have explored the risk factors for in-hospital mortality for HCC patients with PVTT under liver resection.

This study firstly assessed the independent predictive factors for in-hospital mortality in HCC patients with PVTT under surgical resection. In this study, we analyzed 521 of 1531 patients diagnosed of HCC with PVTT and underwent surgical resection in $\mathrm{EHBH}$, which was one of the largest single-center experiences reported in recent years. Among them, $17(3.3 \%)$ patients were dead after liver resection surgery, indicating relatively low in-hospital mortality. The majority of in-hospital death was occurred up to 30 days after surgery $(16 / 17,94.1 \%)$, and the causes of death was all related to severe complications after surgery, such as postoperative massive hemorrhage and acute hepatic failure. Thus, reducing risk factors of these patients could lower the rate of in-hospital death.
In terms of the treatment options for PVTT, type I/II PVTT are more suitable for resection than type III/IV with a significantly longer survival. For type III PVTT patients, as the PVTT has extended to the main portal vein, patients can undergo surgery directly or after tumor downstaging using radiotherapy and/or TACE based on patients' liver function[26, 27]. Further research suggested that patients with type III/IV PVTT and beyond had no significant improvement in survival after surgical treatment[28]. In this study, we founded that type I PVTT patients had a longer survival after surgery, moreover, in-hospital mortality of patients with IV PVTT was remarkably high $(2 / 5,40 \%)$ in this study as well as other studies[10, 17, 19]. Previous studies and our results suggested that the type of PVTT was a guiding indicator for treatment option for HCC patients with PVTT.

The salient contribution of this publication is the identification of certain predictive factors strongly associated with the prognostic outcome of in-hospital mortality of HCC patients with PVTT after surgical resection. So far here have been no widely accepted guidelines on the diagnosis and treatment of HCC with PVTT, and few studies focused on the risk factors [29]. In this study, we firstly proved that total albumin, tumor volume, intraoperative bleeding, and frequency of hospitalization were independent risk factors for in-hospital mortality. Among these predictors, the serum albumin level and tumor volume reflect the conditions of patients' liver function reserve, which were closely related to the postoperative recovery. Previous studies proposed that serum albumin combined with bilirubin values could provide objective and standardized evaluation of remnant liver function, and this approach even showed better discriminatory ability than Child-Pugh class for prognosis in patients with $\mathrm{HCC}[30,31]$. Tumor volume was instructive for evaluating the response of solid tumors to treatment, according to the previous World Health Organization and Response Evaluation Criteria[32]. Emerging imaging techniques, such as new functional imaging and 3-dimensional imaging have been effectively used clinically in recent years to provide a precise tumor volume resection. Our study suggested that patients with a serum albumin level less than $60 \mathrm{~g} / \mathrm{L}$ or tumor volume larger than $50.7 \mathrm{~cm}^{3}$ had a lower survival and higher in-hospital mortality after surgery. This conclusion could provide guidance for what kinds of HCC patients with PVTT are suitable for surgery. Preoperative liver function protection and preoperative imaging to determine tumor volume was necessary to decrease in-hospital mortality. Furthermore, massive intraoperative bleeding has 
been demonstrated to increase the occurrence of post-operative complications and tumor recurrence [33-35]. Intraoperative bleeding should be much concerned as portal veins and hepatic veins vulnerable to injury during surgery of PVTT [36]. Bleeding from major vein could be fatal and extremely difficult to repair during operation. In this study, we also concluded that an intraoperative volume of more than $750 \mathrm{ml}$ might indicate a poor outcome in these patients. In regarding to frequency of hospitalization, it is interesting those HCC patients with PVTT who were in hospitalization at the first time show higher in-hospital mortality. This may be attributed to the fact that first hospitalized patients were performed surgery hastily under inadequate preoperative preparation. In contrast, HCC patients with PVTT who had several times of hospitalization were prepared for operation with a better general statue and liver function. On the whole, preoperative assessment of liver function reserve, reduction of intraoperative bleeding, and multidisciplinary combined diagnosis and treatment could provide HCC patients with PVTT with poor in-hospital mortality and a long-term prognosis.

The limitations of this study should be emphasized: firstly, this single-center study was performed in China which has a high proportion of HBV infection. Secondly, potential bias may exist among the information of 17 dead in hospital patients belong to the insufficient amount and the retrospective nature of the study, which called large sample, multicenter clinical studies focusing on it.

In conclusion, our results indicated that surgery resection produced better survival rate for type I and II PVTT patients instead of type IV PVTT. The treatment for type III should considered the general statue and liver function of HCC patients. As for the risk factors, total albumin, tumor volume or resection volume, intraoperative bleeding and frequency of hospitalization were independent predictive factors for in-hospital mortality in HCC patients with PVTT under surgical resection. Further study is warranted to utilize these factors in promoting early detection, diagnosis, and elective resection to lower in-hospital mortality.

\section{Supplementary Material}

Supplementary figures and tables.

http://www.jcancer.org/v10p0072s1.pdf

\section{Acknowledgements}

This work is supported the Key Project of Natural Science Foundation of China (No: 81730097); the grants of the Science Fund for Creative Research Groups (No:81521091); Chang Jiang Scholars Program
(2013) of China Ministry of Education; the National Key Basic Research Program "973 project" (No: 2015CB554000); Shanghai Municipal Health Bureau (No: SHDC12015106); Shanghai Science and Technology Committee (No: 134119a0200); the National Natural Science Foundation of China (No: 81602523). There are no financial disclosures, conflicts of interest, and/or acknowledgements.

\section{Author Contributions}

Xiu-Ping Zhang and Yu-Zhen Gao: conception of the work, writing manuscript. Shu-Qun Cheng: conception of the work, final approval of manuscript, Zhen-Hua Chen and Yu-Qiang Cheng: analysis and interpretation of data, Wei-Xing Guo and Jie Shi: acquisition of data, Cheng-Qian Zhong and Fan Zhang: acquisition of data.

\section{Competing Interests}

The authors have declared that no competing interest exists.

\section{References}

1. Bruix J, Reig M, Sherman M. Evidence-Based Diagnosis, Staging, and Treatment of Patients With Hepatocellular Carcinoma. Gastroenterology. 2016;150(4):835-53

2. Bruix J, Sherman M, American Association for the Study of Liver D. Management of hepatocellular carcinoma: an update. Hepatology. 2011;53(3):1020-2.

3. Rodriguez-Peralvarez M, Luong TV, Andreana L, Meyer T, Dhillon AP, Burroughs AK. A systematic review of microvascular invasion in hepatocellular carcinoma: diagnostic and prognostic variability. Annals of surgical oncology. 2013;20(1):325-39.

4. Peng ZW, Guo RP, Zhang YJ, Lin XJ, Chen MS, Lau WY. Hepatic resection versus transcatheter arterial chemoembolization for the treatment of hepatocellular carcinoma with portal vein tumor thrombus. Cancer. 2012;118(19):4725-36.

5. Liu PH, Lee YH, Hsia CY, Hsu CY, Huang YH, Chiou YY, et al. Surgical resection versus transarterial chemoembolization for hepatocellular carcinoma with portal vein tumor thrombosis: a propensity score analysis. Annals of surgical oncology. 2014;21(6):1825-33.

6. Schoniger-Hekele M, Muller C, Kutilek M, Oesterreicher C, Ferenci P, Gangl A. Hepatocellular carcinoma in Central Europe: prognostic features and survival. Gut. 2001;48(1):103-9.

7. Forner A, Llovet JM, Bruix J. Hepatocellular carcinoma. Lancet. 2012;379(9822):1245-55

8. Llovet JM, Ricci S, Mazzaferro V, Hilgard P, Gane E, Blanc JF, et al. Sorafenib in advanced hepatocellular carcinoma. The New England journal of medicine. 2008;359(4):378-90.

9. Omata M, Lesmana LA, Tateishi R, Chen PJ, Lin SM, Yoshida H, et al. Asian Pacific Association for the Study of the Liver consensus recommendations on hepatocellular carcinoma. Hepatology international. 2010;4(2):439-74.

10. Wang K, Guo WX, Chen MS, Mao YL, Sun BC, Shi J, et al. Multimodality Treatment for Hepatocellular Carcinoma With Portal Vein Tumor Thrombus: A Large-Scale, Multicenter, Propensity Mathching Score Analysis. Medicine (Baltimore). 2016;95(11):e3015.

11. Shuqun C, Minshan C, Jianqiang C, The National Research Cooperative Group For D, Treatment Of Hepatocellular Carcinoma With Tumor T. Chinese expert consensus on multidisciplinary diagnosis and treatment of hepatocellular carcinoma with portal vein tumor thrombus: 2016 edition. Oncotarget. 2016.

12. Kokudo T, Hasegawa K, Matsuyama Y, Takayama T, Izumi N, Kadoya M, et al. Survival benefit of liver resection for hepatocellular carcinoma associated with portal vein invasion. Journal of hepatology. 2016;65(5):938-43.

13. Sakamoto $K$, Nagano $H$. Surgical treatment for advanced hepatocellular carcinoma with portal vein tumor thrombus. Hepatology research : the official journal of the Japan Society of Hepatology. 2017;47(10):957-62.

14. Shi J, Lai EC, Li N, Guo WX, Xue J, Lau WY, et al. A new classification for hepatocellular carcinoma with portal vein tumor thrombus. Journal of hepato-biliary-pancreatic sciences. 2011;18(1):74-80.

15. Yang T, Lin C, Zhai J, Shi S, Zhu M, Zhu N, et al. Surgical resection for advanced hepatocellular carcinoma according to Barcelona Clinic Liver Cancer (BCLC) staging. Journal of cancer research and clinical oncology. 2012;138(7):1121-9. 
16. Chen J, Huang J, Chen M, Yang K, Chen J, Wang J, et al. Transcatheter arterial chemoembolization (TACE) versus hepatectomy in hepatocellular carcinoma with macrovascular invasion: a meta-analysis of 1683 patients. Journal of Cancer. 2017;8(15):2984-91.

17. Chok KS, Cheung TT, Chan SC, Poon RT, Fan ST, Lo CM. Surgical outcomes in hepatocellular carcinoma patients with portal vein tumor thrombosis. World journal of surgery. 2014;38(2):490-6.

18. Chen JS, Wang Q, Chen XL, Huang XH, Liang LJ, Lei J, et al. Clinicopathologic characteristics and surgical outcomes of hepatocellular carcinoma with portal vein tumor thrombosis. The Journal of surgical research. 2012;175(2):243-50.

19. Ikai I, Hatano E, Hasegawa S, Fujii H, Taura K, Uyama N, et al. Prognostic index for patients with hepatocellular carcinoma combined with tumor thrombosis in the major portal vein. Journal of the American College of Surgeons. 2006;202(3):431-8.

20. Zhang ZM, Lai EC, Zhang C, Yu HW, Liu Z, Wan BJ, et al. The strategies for treating primary hepatocellular carcinoma with portal vein tumor thrombus. International journal of surgery. 2015;20:8-16.

21. Shi J, Lai E, Li N, Guo W, Xue J, Lau W, et al. Surgical treatment of hepatocellular carcinoma with portal vein tumor thrombus. Ann Surg Oncol. 2010;17(8):2073-80.

22. Shi J, Lai EC, Li N, Guo WX, Xue J, Lau WY, et al. Surgical treatment of hepatocellular carcinoma with portal vein tumor thrombus. Annals of surgical oncology. 2010;17(8):2073-80.

23. Xu JF, Liu XY, Wang S, Wen HX. Surgical treatment for hepatocellular carcinoma with portal vein tumor thrombus: a novel classification. World journal of surgical oncology. 2015;13:86.

24. Ye JZ, Zhang YQ, Ye HH, Bai T, Ma L, Xiang BD, et al. Appropriate treatment strategies improve survival of hepatocellular carcinoma patients with portal vein tumor thrombus. World J Gastroenterol. 2014;20(45):17141-7.

25. Zhong JH, Rodriguez AC, Ke Y, Wang YY, Wang L, Li LQ. Hepatic resection as a safe and effective treatment for hepatocellular carcinoma involving a single large tumor, multiple tumors, or macrovascular invasion. Medicine. 2015;94(3):e396.

26. Chen X, Qiu F, Wu Z, Zhang Z, Huang Z, Chen Y, et al. Effects of location and extension of portal vein tumor thrombus on long-term outcomes of surgical treatment for hepatocellular carcinoma. Ann Surg Oncol. 2006;13(7):940-6.

27. Xu J, Liu X, Wang S, Wen H. Surgical treatment for hepatocellular carcinoma with portal vein tumor thrombus: a novel classification. World J Surg Oncol. 2015;13:86.

28. Kokudo T, Hasegawa K, Matsuyama Y, Takayama T, Izumi N, Kadoya M, et al. Survival benefit of liver resection for hepatocellular carcinoma associated with portal vein invasion. J Hepatol. 2016;65(5):938-43.

29. Cheng S, Chen M, Cai J. Chinese expert consensus on multidisciplinary diagnosis and treatment of hepatocellular carcinoma with portal vein tumor thrombus: 2016 edition. Oncotarget. 2017;8(5):8867-76.

30. Yoh T, Seo S, Ogiso S, Kawai T, Okuda Y, Ishii T, et al. Proposal of a New Preoperative Prognostic Model for Solitary Hepatocellular Carcinoma Incorporating 18F-FDG-PET Imaging with the ALBI Grade. Ann Surg Oncol. 2017.

31. Ogasawara S, Chiba T, Ooka Y, Suzuki E, Kanogawa N, Saito T, et al. Liver function assessment according to the Albumin-Bilirubin (ALBI) grade in sorafenib-treated patients with advanced hepatocellular carcinoma. Invest New Drugs. 2015;33(6):1257-62.

32. Taylor P, Haverstick D. Re: New guidelines to evaluate the response to treatment in solid tumors (ovarian cancer). J Natl Cancer Inst. 2005;97(2):151; author reply 2 .

33. Quesada R, Poves I, Berjano E, Vilaplana C, Andaluz A, Moll X, et al. Impact of monopolar radiofrequency coagulation on intraoperative blood loss during liver resection: a prospective randomised controlled trial. International journal of hyperthermia : the official journal of European Society for Hyperthermic Oncology, North American Hyperthermia Group. 2016:1-7.

34. Nakajima $Y$, Shimamura $T$, Kamiyama $T$, Matsushita M, Sato N, Todo S. Control of intraoperative bleeding during liver resection: analysis of a questionnaire sent to 231 Japanese hospitals. Surgery today. 2002;32(1):48-52.

35. Miyazawa $M$, Aikawa $M$, Okada $K$, Watanabe $\mathrm{Y}$, Okamoto $\mathrm{K}$, Koyama I. Laparoscopic liver resection using a monopolar soft-coagulation device to provide maximum intraoperative bleeding control for the treatment of hepatocellular carcinoma. Surgical endoscopy. 2017.

36. Gupta R, Fuks D, Bourdeaux C, Radkani P, Nomi T, Lamer C, et al. Impact of intraoperative blood loss on the short-term outcomes of laparoscopic liver resection. Surgical endoscopy. 2017;31(11):4451-7. 